\title{
Optimized Motion Strategies for Localization in Leader-Follower Formations
}

\author{
Xun S. Zhou, Ke X. Zhou, and Stergios I. Roumeliotis
}

\begin{abstract}
This paper addresses the problem of determining the optimal robot trajectory for localizing a robot follower in a leader-follower formation using robot-to-robot distance or bearing measurements. In particular, maintaining a perfect formation has been shown to reduce the localization accuracy (as compared to moving randomly), or even leads to loss of observability when only distance or bearing measurements are available and the robots move on parallel straight lines. To address this limitation, we allow the follower to slightly deviate from its desired formation-imposed position and seek to find the next best location where it should move to in order to minimize the uncertainty about its relative, with respect to the leader, position and orientation estimates. We formulate and solve this non-convex optimization problem analytically and show, through extensive simulations, that the proposed optimized motion strategy leads to significant localization accuracy improvement as compared to competing approaches.
\end{abstract}

\section{INTRODUCTION}

Robots are often required to move in a formation. For example, in military missions, aerial vehicles fly in formations for mutual defense and reconnaissance; in transportation, vehicle platooning saves fuel and increases the throughput of transportation networks [1]; and in cooperative object manipulation tasks, multiple robots form a formation in order to move a large payload [2].

Due to its broad applications, formation control has been extensively studied, and there exist many approaches for maintaining formation, such as behavior-based [3], virtual structure [4], and leader-follower-based [5] methods. All these methods rely on on-board sensors to provide information about the robots' relative positions and orientations (poses), so as to reduce the deviations from the desired poses. However, sensors usually do not measure the relative pose directly, but only measure the range or bearing from one robot to its neighbors. Therefore, an estimator is often employed for estimating the relative pose using inter-robot measurements. Clearly, successful formation control requires accurate relative pose estimates.

Interestingly, robot motion affects the accuracy of relative pose estimates. Early experimental results of Rekleitis et al. [6] show that random robot motion improves the accuracy of the relative pose estimates as compared to moving in a formation. In addition, Trawny and Barfoot [7] also find that the optimal motion strategy is not to move in a formation.

The estimation accuracy in robot formations degrades for two reasons. Firstly, the multi-robot system with distance or

This work was supported by the University of Minnesota (DTC), and the National Science Foundation (IIS-0643680, IIS-0811946, IIS-0835637).

X. S. Zhou, and S. I. Roumeliotis are with the Department of Computer Science and Engineering, University of Minnesota, Minneapolis, MN 55455, USA $\{$ zhou, stergios $\}$ @ Cs. umn. edu

K. X. Zhou is with the Department of Electrical and Computer Engineering, University of Minnesota, Minneapolis, MN 55455, USA kezhoudece.umn. edu

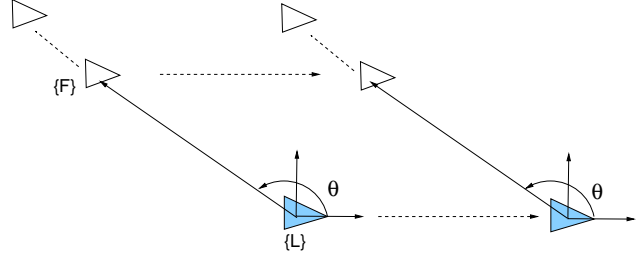

Fig. 1. A leader, L, and a follower, F, are moving on parallel straight lines. The relative pose between the leader and follower are unobservable when the robots only measure the bearing $\theta$, because the follower can be anywhere along the line of sight of the leader.

bearing measurements is non-linear, hence, the information gained from the measurements depends on the system's state (relative robot poses). A constant system state usually does not provide the maximum information. Hence, the robot formation is often inferior to randomized robot trajectories. Secondly, the system is unobservable when the robots move in formation and observe only the range or bearing between robots, because their relative poses do not change, and range or bearing measurements of a constant state do not provide sufficient information to determine the robots' relative poses (see Fig. 1). However, the system becomes observable when the robots move arbitrarily [8]-[10], because knowledge of the robot motions provides additional information about their relative poses. As a result, random trajectories show better performance than moving in formation. Hence, there is a trade-off between keeping the formation and improving the estimation accuracy.

To analyze this trade-off, we seek the optimal next-time step follower position in a leader-follower formation. In order to balance the requirement of moving in formation and improving the estimation accuracy, the follower is allowed to move away from the desired position within a given range. The specific estimator employed in this work is the Extended Kalman Filter (EKF), and the optimization criterion used is the weighted trace of the posterior covariance matrix. Our main contribution is that we find the global optimal solution of this optimization problem analytically by first computing all critical points (i.e., the points which satisfy the KarushKuhn-Tucker (KKT) necessary optimality conditions) and select as global minimum the one that yields the lowest cost.

The remainder of the paper is organized as follows. In the following section, we describe related localization approaches in robot formation. In Section III, we formulate the optimal motion strategy problem and present our solution in Section IV followed by simulation results (Section V). Finally, in Section VI, we draw our conclusions and suggest future research directions. 


\section{RELATED WORK}

Current research on localization in mobile robot formations mainly focuses on designing specific formation patterns that yield the highest localization accuracy for the team. Specifically, Zhang et al. [11] study the impact of the robots spatial configuration on the localization accuracy for static robots that receive absolute position measurements, as well as robot-to-robot range and/or bearing measurements. They establish the necessary and sufficient conditions for the team of robots to be completely localized, and show that the covariance matrix is a function of the relative robot poses. A robot formation that minimizes the trace of the covariance matrix is determined by a gradient descent optimization technique. However, due to the nonconvexity of the objective function, the chosen optimization algorithm does not guarantee global optimality.

Kurazume and Hirose [12] propose a leapfrogging strategy for a team of robots comprised with one master and two slave robots. The master and slave robots alternate in moving forward on straight paths toward a destination, while the slave robots act as portable landmarks for localizing the master robot. The authors study the effect of the master-slave relative position on localization accuracy, and find three configurations that show superior performance, by numerically minimizing a weighted least squares cost function. However, the requirement of at least one robot to remain stationary is undesirable.

In [7], all robots move continuously toward a target configuration while measuring distance to each other. In order to determine the optimal trajectories, the authors employ a gradient-based optimization algorithm to minimize the determinant of the covariance matrix at the target configuration. Even though the gradient-based algorithm can only converge to a local optimum, their numerical experiments indicate that positioning accuracy is improved when the robots do not move in formation.

The effects of formation geometry on the localization accuracy is studied in [13], where the robots record relative position measurements, and the robots' orientations are assumed perfectly known. The optimality criterion is the steady-state position uncertainty of the robot team. A genetic algorithm is employed for determining the optimal relative robot positions. This algorithm is shown to be a suitable tool for the problem because of the existence of multiple local minima in the objective function. Their results indicate that the optimal formation geometry comprises of adjacent equilateral triangles.

The papers mentioned above have focused on observable systems, where the robots have sufficient measurements (e.g., relative position, orientation, and absolution position) for determining their relative poses. However, due to cost considerations in practice, the robots may only have access to a limited type of measurements, such as inter-robot range or bearing. In this case, the relative poses are unobservable when the robots move in formation on straight lines, and the trade-off between maintaining the formation and system observability has generally been overlooked with one exception. Mariottini et al. [10] study the problem of loss

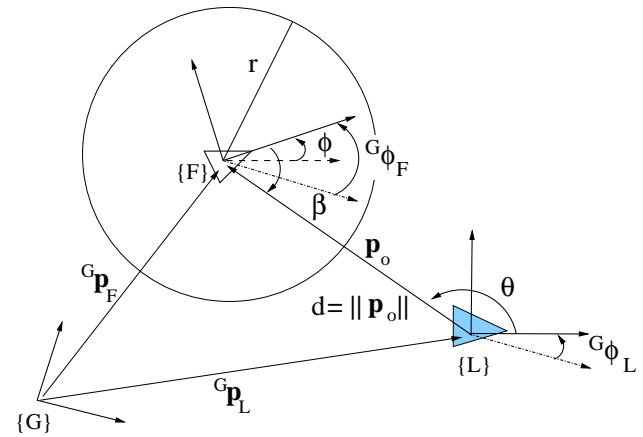

Fig. 2. A leader-follower formation: the follower $\mathrm{F}$ is at the desired position $\mathbf{p}_{o}$ with respect to the leader L. The follower measures either the distance $d$, or bearing $\beta$ to the leader. In order to gain observability and minimize the relative pose estimation error, the follower is allowed to move inside the circle centered at $\mathbf{p}_{o}$ with radius $r$.

of observability in robot formations with bearing-only measurements, and propose a switching active-sensing strategy to maintain observability and the formation. Specifically, a standard leader-follower controller drives the follower to the desired position when the leader does not move on straight paths. When the system becomes unobservable, the standard controller is switched to an active-formation controller that drives the follower to a position which is slightly different from the desired position. However, no optimality, in terms of the estimation accuracy, is claimed by the active controller.

Our work differs from the aforementioned papers, in that our objective is to balance the trade-off between maintaining the formation and improving the estimation accuracy, while analytically computing the optimal solution.

\section{PROBLEM Formulation}

In a leader-follower formation, the leader moves on a desired trajectory, while the follower keeps its position $\mathbf{p}=$ $\left[\begin{array}{ll}x & y\end{array}\right]^{T}$ and orientation $\phi$ constant with respect to the leader ${ }^{1}$. We define the state vector as the follower's position and orientation with respect to the leader, $\mathbf{x}=[x, y, \phi]^{T}$, and denote the desired follower state as $\mathbf{x}_{o}=\left[\begin{array}{lll}x_{o}, & y_{o}, & \phi_{o}\end{array}\right]^{T}$. Furthermore, the follower measures the distance $d$, or bearing $\beta$ to the leader (see Fig. 2). The follower estimates its state using these measurements, so as to maintain the formation. Due to the trade-off between maintaining the formation and the estimation accuracy discussed before, we allow the follower's position to deviate from the desired position within a given distance $r$. Our objective is to determine where the follower should be at the next time step to record a relative measurement, such that the estimate uncertainty is minimized.

In the following sections, we will first present the system dynamic model $^{2}$, the measurement model, and then formulate the optimization problem that needs to be solved.

\footnotetext{
${ }^{1}$ In this paper, we only consider the case with one follower. The same algorithm can be applied to the case of multiple followers.

${ }^{2}$ Note that our optimization framework can be applied with any system dynamic model. The specific model presented is used in our simulations to evaluate the performance of our method.
} 


\section{A. System Kinematic Model}

We assume that both the leader and the follower are nonholonomic vehicles whose linear and rotational velocities are $\left(v_{L}, \omega_{L}\right)$ and $\left(v_{F}, \omega_{F}\right)$, respectively. In addition, we focus on the case where the leader is moving on a straight line with constant $v_{L}$, and $\omega_{L}=0$, because, most likely, it is the dominant motion when a team of robots are moving towards a distant destination. The directions of the linear velocities are along the $x$-axes of the vehicles' body frames. Hence, these linear velocities expressed in a fixed global frame $\{G\}$ are described by

$$
{ }^{G} \dot{\mathbf{p}}_{L}={ }_{L}^{G} \mathbf{C} \cdot \mathbf{e}_{1} v_{L}, \quad{ }^{G} \dot{\mathbf{p}}_{F}={ }_{F}^{G} \mathbf{C} \cdot \mathbf{e}_{1} v_{F} .
$$

where $\mathbf{e}_{1}=\left[\begin{array}{ll}1 & 0\end{array}\right]^{T},{ }_{L}^{G} \mathbf{C}$ and ${ }_{F}^{G} \mathbf{C}$ are the rotation matrices that project vectors expressed in frame $\{L\}$ and $\{F\}$ to frame $\{G\}$, respectively.

The rotation matrices ${ }_{L}^{G} \mathbf{C},{ }_{F}^{G} \mathbf{C}$, and ${ }_{F}^{L} \mathbf{C}$ satisfy the following relationship:

$$
\begin{aligned}
{ }_{F}^{G} \mathbf{C}\left({ }^{G} \phi_{F}\right) & ={ }_{L}^{G} \mathbf{C}\left({ }^{G} \phi_{L}\right) \cdot{ }_{F}^{L} \mathbf{C}(\phi) \\
\Rightarrow{ }^{G} \phi_{F} & ={ }^{G} \phi_{L}+\phi .
\end{aligned}
$$

Differentiating (3) with respect to time, yields the dynamic model for the orientation:

$$
\dot{\phi}={ }^{G} \dot{\phi}_{F}-{ }^{G} \dot{\phi}_{L}=\omega_{F}-\omega_{L}=\omega_{F} .
$$

Similarly, we derive the dynamic model for the robots' relative position $\mathbf{p}$ by taking the time derivative of the following geometric constraint

$$
\begin{aligned}
\mathbf{p} & ={ }_{L}^{G} \mathbf{C}^{T}\left({ }^{G} \mathbf{p}_{F}-{ }^{G} \mathbf{p}_{L}\right) \\
\Rightarrow \dot{\mathbf{p}} & ={ }_{L}^{G} \mathbf{C}^{T}\left({ }^{G} \dot{\mathbf{p}}_{F}-{ }^{G} \dot{\mathbf{p}}_{L}\right) .
\end{aligned}
$$

Substituting (1) and (2) in (6), we obtain

$$
\dot{\mathbf{p}}={ }_{F}^{L} \mathbf{C} \cdot \mathbf{e}_{1} v_{F}-\mathbf{e}_{1} v_{L}
$$

Rearranging terms in (4) and (7), yields the following continuous-time dynamic model:

$$
\begin{aligned}
& \dot{x}=\cos (\phi) v_{F}-v_{L} \\
& \dot{y}=\sin (\phi) v_{F} \\
& \dot{\phi}=\omega_{F} .
\end{aligned}
$$

\section{B. State and Covariance Propagation}

The discrete-time kinematic equations of the follower's pose are obtained by discretizing (8) using Euler's forward method.

$$
\begin{aligned}
x_{k+1} & =x_{k}+\cos \left(\phi_{k}\right) v_{F_{k}} \delta t-v_{L} \delta t \\
y_{k+1} & =y_{k}+\sin \left(\phi_{k}\right) v_{F_{k}} \delta t \\
\phi_{k+1} & =\phi_{k}+\omega_{F_{k}} \delta t .
\end{aligned}
$$

We employ the EKF to propagate the estimated follower's pose $^{3}$ using the control inputs (odometry measurements) $v_{F_{m k}}$ and $\omega_{F_{m k}}$

$$
\begin{aligned}
\hat{x}_{k+1 \mid k} & =\hat{x}_{k \mid k}+\cos \left(\hat{\phi}_{k \mid k}\right) v_{F_{m k}} \delta t-v_{L} \delta t \\
\hat{y}_{k+1 \mid k} & =\hat{y}_{k \mid k}+\sin \left(\hat{\phi}_{k \mid k}\right) v_{F_{m k}} \delta t \\
\hat{\phi}_{k+1 \mid k} & =\hat{\phi}_{k \mid k}+\omega_{F_{m k}} \delta t .
\end{aligned}
$$

\footnotetext{
${ }^{3}$ In the rest of the paper, the "hat" symbol "^" denotes the estimated value of a quantity. The subscript $l \mid j$ refers to the estimate of the quantity at time-step $l$ after incorporating all measurements up to time-step $j$. The error between the actual value of the quantity and its estimate is denoted by the "tilde" symbol " $"$ ". The relationship between the actual value $x$ and the estimate $\hat{x}$ is $\tilde{x}=x-\hat{x}$.
}

By linearizing (10), the error-propagation equation for the follower's pose is readily derived as:

$$
\begin{aligned}
{\left[\begin{array}{c}
\tilde{x}_{k+1 \mid k} \\
\tilde{y}_{k+1 \mid k} \\
\tilde{\phi}_{k+1 \mid k}
\end{array}\right]=} & {\left[\begin{array}{ccc}
1 & 0 & -\sin \left(\hat{\phi}_{k \mid k}\right) v_{F_{m k}} \delta t \\
0 & 1 & \cos \left(\hat{\phi}_{k \mid k}\right) v_{F_{m k}} \delta t \\
0 & 0 & 1
\end{array}\right]\left[\begin{array}{c}
\tilde{x}_{k \mid k} \\
\tilde{y}_{k \mid k} \\
\tilde{\phi}_{k \mid k}
\end{array}\right] } \\
+ & {\left[\begin{array}{cc}
\cos \left(\hat{\phi}_{k \mid k}\right) \delta t & 0 \\
\sin \left(\hat{\phi}_{k \mid k}\right) \delta t & 0 \\
0 & \delta t
\end{array}\right]\left[\begin{array}{c}
w_{v_{F_{k}}} \\
w_{\omega_{F_{k}}}
\end{array}\right] } \\
\Leftrightarrow \tilde{\mathbf{x}}_{k+1 \mid k}= & \mathbf{\Phi}_{k} \tilde{\mathbf{x}}_{k \mid k}+\mathbf{G}_{k} \mathbf{w}_{k}
\end{aligned}
$$

where $w_{v_{F_{k}}}$ and $w_{\omega_{F_{k}}}$ are white, zero-mean, Gaussian noise sequences with variances $\sigma_{v_{F_{k}}}^{2}$ and $\sigma_{\omega_{F_{k}}}^{2}$, affecting the linear and rotational velocity inputs, respectively.

From equation (11), the error-state covariance propagation equation can be written as:

$$
\mathbf{P}_{k+1 \mid k}=\boldsymbol{\Phi}_{k} \mathbf{P}_{k \mid k} \boldsymbol{\Phi}_{k}^{T}+\mathbf{G}_{k} \mathbf{Q}_{k} \mathbf{G}_{k}^{T}
$$

where $\mathbf{Q}_{k}=\operatorname{diag}\left(\sigma_{v_{F_{k}}}^{2}, \sigma_{\omega_{F_{k}}}^{2}\right)$.

\section{Measurement Update}

The follower employs the inter-robot measurements to perform pose updates in the EKF. The inter-robot measurement at time step $k$ can be described by the generally nonlinear model

$$
z_{k+1}=h\left(\mathbf{x}_{k+1}, n_{k+1}\right)
$$

where $n_{k+1}$ is the Gaussian noise with covariance $\mathbf{R}_{k+1}$. In the EKF, we employ linearization to obtain the measurement error equation

$$
\tilde{z}_{k+1}=\mathbf{H}_{k+1} \tilde{\mathbf{x}}_{k+1 \mid k}+\Gamma_{k+1} n_{k+1}
$$

where

$$
\begin{aligned}
\mathbf{H}_{k+1} & =\left.\nabla_{\mathbf{x}_{k+1}} h\left(\mathbf{x}_{k+1}, n_{k+1}\right)\right|_{\hat{\mathbf{x}}_{k+1 \mid k}, 0} \\
\Gamma_{k+1} & =\left.\nabla_{n_{k+1}} h\left(\mathbf{x}_{k+1}, n_{k+1}\right)\right|_{\hat{\mathbf{x}}_{k+1 \mid k}, 0} .
\end{aligned}
$$

The Jacobian matrices $\mathbf{H}_{k+1}$ and $\Gamma_{k+1}$ are time varying and depend on the state estimates. Thus, the location where the robot records the measurement will affect the accuracy of the state estimates. In this paper, we consider two types of inter-robot measurements, namely the inter-robot range, and bearing.

1) Range measurements: The relative range measurement between the leader and the follower is described by the following model (see Fig 2):

$$
z_{k+1}=d_{k+1}+n_{d_{k+1}}=\sqrt{x_{k+1}^{2}+y_{k+1}^{2}}+n_{d_{k+1}}
$$

where the noise $n_{d_{k+1}}$ is zero-mean Gaussian process with variance $\mathbf{R}_{d}=\sigma_{d}^{2}$.

The measurement Jacobian for the distance measurement is a $1 \times 3$ vector:

$$
\begin{aligned}
\mathbf{h}_{d_{k+1}} & =\left[\begin{array}{lll}
\frac{\hat{x}_{k+1 \mid k}}{\hat{d}_{k+1 \mid k}} & \frac{\hat{y}_{k+1 \mid k}}{\hat{d}_{k+1 \mid k}} & 0
\end{array}\right] \\
& =\left[\begin{array}{lll}
\cos \left(\hat{\theta}_{k+1 \mid k}\right) & \sin \left(\hat{\theta}_{k+1 \mid k}\right) & 0
\end{array}\right]
\end{aligned}
$$

where $\hat{d}_{k+1 \mid k}=\sqrt{\hat{x}_{k+1 \mid k}^{2}+\hat{y}_{k+1 \mid k}^{2}}$. 
2) Bearing measurements: The relative bearing from the follower to the leader is described by (see Fig 2):

$$
\begin{aligned}
z_{k+1} & =\beta_{k+1}+n_{\beta_{k+1}}=\theta_{k+1}-\phi_{k+1}+\pi+n_{\beta_{k+1}} \\
& =\arctan \left(\frac{y_{k+1}}{x_{k+1}}\right)-\phi_{k+1}+\pi+n_{\beta_{k+1}}
\end{aligned}
$$

where $n_{\beta_{k+1}}$ is zero-mean Gaussian noise with variance $\mathbf{R}_{\beta}=\sigma_{\beta}^{2}$.

The measurement Jacobian for the bearing measurement has the following structure:

$$
\begin{aligned}
\mathbf{h}_{\beta_{k+1}} & =\left[\begin{array}{lll}
-\frac{\hat{y}_{k+1 \mid k}}{\hat{d}_{k+1 \mid k}^{2}} & \frac{\hat{x}_{k+1 \mid k}}{\hat{d}_{k+1 \mid k}^{2}} & -1
\end{array}\right] \\
& =\frac{1}{\hat{d}_{k+1 \mid k}}\left[\begin{array}{lll}
-\sin \left(\hat{\theta}_{k+1 \mid k}\right) & \cos \left(\hat{\theta}_{k+1 \mid k}\right) & -\hat{d}_{k+1 \mid k}
\end{array}\right] .
\end{aligned}
$$

3) Posterior covariance: The inter-robot measurements are processed by the EKF to update the follower's pose estimate. The covariance update equation is

$$
\mathbf{P}_{k+1 \mid k+1}=\mathbf{P}_{k+1 \mid k}-\mathbf{P}_{k+1 \mid k} \mathbf{H}_{k+1}^{T} \mathbf{S}_{k+1}^{-1} \mathbf{H}_{k+1} \mathbf{P}_{k+1 \mid k}
$$

where $\mathbf{S}_{k+1}=\mathbf{H}_{k+1} \mathbf{P}_{k+1 \mid k} \mathbf{H}_{k+1}^{T}+\mathbf{R}_{k+1}$. In this equation, $\mathbf{H}_{k+1}$ and $\mathbf{R}_{k+1}$ are the measurement Jacobian matrix and noise covariance matrix at time-step $k+1$, respectively. The posterior covariance indicates the accuracy of the estimate and it is a function of the propagated state. However, the actual value of the measurement at the next time step is not required for computing the posterior covariance, therefore it is possible to optimize the robot motion to achieve higher accuracy. In the next section, we will formulate the robot motion strategy as an optimization problem. The optimization variable is the next time-step position $\hat{\mathbf{p}}_{k+1 \mid k}$.

\section{Optimization Problem Formulation}

The cost function is defined as the weighted sum of the diagonal elements of the posterior covariance,

$$
c=\operatorname{tr}\left(\mathbf{W} \mathbf{P}_{k+1 \mid k+1} \mathbf{W}\right)
$$

where the weighting matrix $\mathbf{W}=\operatorname{diag}\left(1,1, d_{o}\right)$ with $d_{o}=$ $\left\|\mathbf{p}_{o}\right\|_{2}$. Since $\boldsymbol{\Phi}_{k}$ [see (11)] is a function of the control input $v_{F_{m k}}$, the prior covariance $\mathbf{P}_{k+1 \mid k}$ [see (12)] is also a function of the optimization variable $\hat{\mathbf{p}}_{k+1 \mid k}$, which makes solving the optimization problem very challenging. However, since the robots are required to move in formation, the follower can only deviate from the nominal position $\mathbf{p}_{o}$ by a small distance $r$. So we approximate the prior covariance by a constant matrix $\mathbf{P}$ which is chosen to be the prior covariance when the follower moves to the desired position $\mathbf{p}_{o}$. From here on, we will drop the subscripts for the state, covariance, and measurement Jacobian, when the meaning of each quantity is clear in the context to simplify our notation, e.g., $\mathbf{p}=\hat{\mathbf{p}}_{k+1 \mid k}$ and $\mathbf{P}=\mathbf{P}_{k+1 \mid k}$.

The objective function can be simplified by

$$
\begin{aligned}
& \underset{\mathbf{p}}{\operatorname{argmin}} \operatorname{tr}\left(\mathbf{W} \mathbf{P}_{k+1 \mid k+1} \mathbf{W}\right) \\
&=\underset{\mathbf{p}}{\operatorname{argmin}}-\operatorname{tr}\left(\mathbf{W} \mathbf{P} \mathbf{H}^{T} \mathbf{S}^{-1} \mathbf{H P W}\right) .
\end{aligned}
$$

Defining $f=-\operatorname{tr}\left(\mathbf{W} \mathbf{P} \mathbf{H}^{T} \mathbf{S}^{-1} \mathbf{H P W}\right)$, we formulate the formation optimization problem as

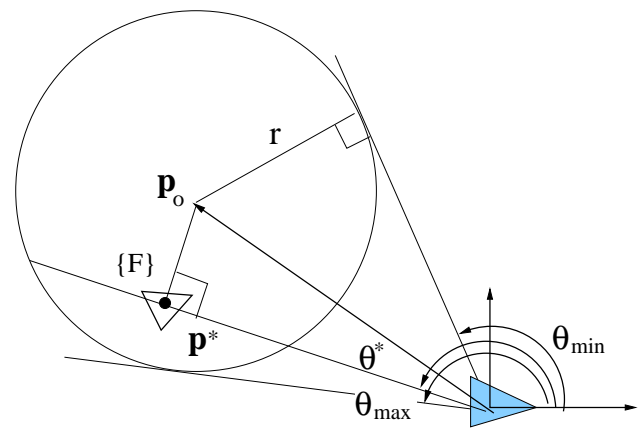

$\{\mathrm{L}\}$

Fig. 3. Optimal solution for the distance-only case. Since the measurement Jacobian $\mathbf{h}_{d}$ is a function of only $\theta$, the cost function values are the same for all points on the line with angle $\theta$. From the optimal solution $\theta^{*} \in\left[\theta_{\min }\right.$, $\left.\theta_{\max }\right]$, we determine the optimal next step position $\mathbf{p}^{*}$ as the nearest point to $\mathbf{p}_{o}$.

\section{- Optimization Problem $1\left(\Pi_{1}\right)$}

$$
\begin{aligned}
\underset{\mathbf{p}}{\min } . & f \\
\text { s.t. } & g=\left\|\mathbf{p}-\mathbf{p}_{o}\right\|_{2}-r \leq 0
\end{aligned}
$$

Remark 1: The optimization problem $\Pi_{1}$ is a nonlinear programming problem since both the objective function $f$ and the constraint $g$ are nonlinear functions of the optimization variable. Furthermore, $\Pi_{1}$ is not a convex problem, because the objective function is not convex.

\section{Problem Solution}

As mentioned in the previous section, the optimization problem $\Pi_{1}$ is not convex, and thus it has multiple local minima. A naive approach is to discretize the feasible set and find the point which yields the minimum value for the function $f$. However, such an approach provides no guarantees for finding the global optimum, due to limitations of the grid resolution.

Instead, we find the global optimal solution as follows: We first determine all critical/stationary points (i.e., those points which satisfy the Karush-Kuhn-Tucker (KKT) necessary optimality conditions [14, Ch. 3]) analytically and evaluate their objective values. Then, as optimal solution, we select the critical point whose objective value is the smallest.

\section{A. Distance-only}

For the distance-only case, the measurement Jacobian $\mathbf{h}_{d}$ [see (16)] only depends on the bearing angle $\theta$ from the leader to the follower, hence the objective function $f$ also only depends on $\theta$. Therefore, we change the optimization variable from the position $\mathbf{p}$ to the bearing angle $\theta$, and reformulate problem $\Pi_{1}$ to the optimization problem $\Pi_{2}$.

- Optimization Problem $2\left(\Pi_{2}\right)$

$$
\begin{array}{cl}
\min _{\theta} & f_{d}(\theta) \\
\text { s.t. } & \theta_{\min } \leq \theta \leq \theta_{\max }
\end{array}
$$

where $\theta_{\min }$ is the minimum and $\theta_{\max }$ is the maximum bearing angle that satisfies the constraint $g$. From the optimal solution $\theta^{*}$ of problem $\Pi_{2}$, we choose the optimal next step position $\mathbf{p}^{*}$ as the nearest point on the line to $\mathbf{p}_{o}$ (see Fig. 3). 
The global optimal solution $\theta^{*}$ can appear in the following two cases: (i) the constraint is inactive, then $\theta^{*}$ satisfies $\nabla_{\theta} f_{d}(\theta)=0$; (ii) the constraint is active, then $\theta^{*}=\theta_{\text {min }}$, or $\theta^{*}=\theta_{\max }$. We examine both cases, and determine the global optimal solution $\theta^{*}$ by searching for the one with the smallest objective value. In the following, we present our solution for $\nabla_{\theta} f_{d}(\theta)=0$.

The objective function $f_{d}(\theta)$ is a fractional function whose numerator and denominator are both linear in $\sin (2 \theta)$ and $\cos (2 \theta)$.

$$
\begin{aligned}
f_{d}(\theta) & =-\frac{u}{v}=-\frac{\operatorname{tr}\left(\mathbf{W} \mathbf{P} \mathbf{h}_{d}^{T} \mathbf{h}_{d} \mathbf{P} \mathbf{W}\right)}{\mathbf{h}_{d} \mathbf{P} \mathbf{h}_{d}^{T}+\mathbf{R}_{d}} \\
& =-\frac{a_{1} \sin (2 \theta)+a_{2} \cos (2 \theta)+a_{3}}{a_{4} \sin (2 \theta)+a_{5} \cos (2 \theta)+a_{6}},
\end{aligned}
$$

where the prior covariance is a constant matrix with elements:

$$
\mathbf{P}=\left[\begin{array}{lll}
p_{1} & p_{4} & p_{5} \\
p_{4} & p_{2} & p_{6} \\
p_{5} & p_{6} & p_{3}
\end{array}\right]
$$

and

$$
\begin{aligned}
& a_{1}=p_{1} p_{4}+p_{2} p_{4}+d_{o}^{4} p_{5} p_{6} \\
& a_{2}=\left(p_{1}^{2}-p_{2}^{2}+d_{o}^{4} p_{5}-d_{o}^{4} p_{6}\right) / 2 \\
& a_{3}=\left(p_{1}^{2}+p_{2}^{2}+2 p_{4}^{2}+d_{o}^{4} p_{5}^{2}+d_{o}^{4} p_{6}^{2}\right) / 2 \\
& a_{4}=p_{4}, a_{5}=\left(p_{1}-p_{2}\right) / 2, a_{6}=\left(p_{1}+p_{2}\right) / 2+\sigma_{d}^{2} .
\end{aligned}
$$

Using the quotient rule, the gradient of the objective function $f_{d}$ is

$\nabla_{\theta} f_{d}=-\frac{u^{\prime} v-u v^{\prime}}{v^{2}}=-\frac{2\left(\sqrt{b_{1}^{2}+b_{2}^{2}} \sin (2 \theta+\varphi)+b_{3}\right)}{v^{2}}$, where

$$
\begin{aligned}
& b_{1}=a_{3} a_{5}-a_{2} a_{6}, b_{2}=a_{1} a_{6}-a_{3} a_{4} \\
& b_{3}=a_{1} a_{5}-a_{2} a_{4}, \varphi=\operatorname{atan} 2\left(b_{2}, b_{1}\right) .
\end{aligned}
$$

Since the denominator $v$ is always nonzero, $\nabla_{\theta} f_{d}(\theta)=0$ is equivalent to

$$
\sqrt{b_{1}^{2}+b_{2}^{2}} \sin (2 \theta+\varphi)+b_{3}=0 .
$$

For $\theta \in(-\pi, \pi]$, there are four critical points, two of which are the minima:

$$
\theta_{1}^{*}=\frac{\pi-\alpha-\varphi}{2}, \quad \theta_{2}^{*}=\frac{-\pi-\alpha-\varphi}{2}
$$

where $\alpha=\arcsin \left(-\frac{b_{3}}{\sqrt{b_{1}^{2}+b_{2}^{2}}}\right)$.

If $\theta_{1}^{*}$ or $\theta_{2}^{*}$ satisfies the constraint (23), then the optimal solution is $\theta_{1}^{*}$ or $\theta_{2}^{*}$. Otherwise, the optimal solution is $\theta_{\min }$ or $\theta_{\max }$ (both of which can be easily computed from the geometry of Fig. 3) whichever yields a smaller value for the objective function.

\section{B. Bearing-only}

For the bearing-only case, the measurement Jacobian $\mathbf{h}_{\beta}$ [see (18)] depends on both $\theta$ and $d$. Therefore, contrary to the distance-only case, the objective function for the bearingonly case is an explicit function of both $d$ and $\theta$. Using the same parametrization employed in the distance-only case, we express the optimization variable $\mathbf{p}$ using polar coordinates, i.e., $\mathbf{p}:=d[\cos (\theta) \sin (\theta)]^{T}$. Transforming the optimization problem $\Pi_{1}$ for the bearing-only case into polar coordinates, results in the following problem

- Optimization Problem $3\left(\Pi_{3}\right)$

$$
\begin{aligned}
\min _{d, \theta} & f_{\beta}=-\frac{c_{u} d^{2}+2 \mathbf{b}_{u}^{T} \boldsymbol{\alpha} d+\boldsymbol{\alpha}^{T} \mathbf{A}_{u} \boldsymbol{\alpha}}{c_{v} d^{2}+2 \mathbf{b}_{v}^{T} \boldsymbol{\alpha} d+\boldsymbol{\alpha}^{T} \mathbf{A}_{v} \boldsymbol{\alpha}} \\
\text { s.t. } & g_{\beta}=\left(d \cos (\theta)-x_{o}\right)^{2}+\left(d \sin (\theta)-y_{o}\right)^{2}-r^{2} \leq 0,
\end{aligned}
$$

where $\boldsymbol{\alpha}=[-\sin (\theta) \cos (\theta)]^{T}$ and $\mathbf{A}_{u}, \mathbf{b}_{u}, c_{u}, \mathbf{A}_{v}, \mathbf{b}_{v}$, and $c_{v}$ are known parameters expressed in terms of $\mathbf{P}, d_{o}$, and $\sigma_{\beta}^{2}$ (see [15] for their specific expressions).

In order to solve $\Pi_{3}$, we proceed as follows: We first determine all critical/stationary points by solving systems of polynomial equations using an elimination procedure, and evaluate their objective values. Then, as optimal solution for $\Pi_{3}$ we select the critical point whose objective value is the smallest.

To proceed, we first construct the Lagrange function [14]

$$
\mathcal{L}(d, \theta, \lambda)=f_{\beta}(d, \theta)+\lambda g_{\beta}(d, \theta) .
$$

Based on the KKT necessary conditions, the critical points $d^{*}, \theta^{*}$, and the associated Lagrange multiplier $\lambda^{*}$ must satisfy

$$
\begin{aligned}
\nabla f_{\beta}\left(d^{*}, \theta^{*}\right)+\lambda^{*} \nabla g_{\beta}\left(d^{*}, \theta^{*}\right) & =\mathbf{0}_{2 \times 1} \\
\lambda^{*} g_{\beta}\left(d^{*}, \theta^{*}\right) & =0 \\
\lambda^{*} & \geq 0,
\end{aligned}
$$

where $\nabla f_{\beta}=\left[\begin{array}{ll}\nabla_{d} f_{\beta} & \nabla_{\theta} f_{\beta}\end{array}\right]^{T}$ and $\nabla g_{\beta}=\left[\begin{array}{lll}\nabla_{d} g_{\beta} & \nabla_{\theta} g_{\beta}\end{array}\right]^{T}$. As shown in [15], both $f_{\beta}$ and its derivative $\nabla f_{\beta}$ are rational functions with respect to $d^{*}, \cos \left(\theta^{*}\right)$, and $\sin \left(\theta^{*}\right)$, while $g_{\beta}$ and $\nabla g_{\beta}$ are polynomials in those three variables. Thus, (28) can be transformed into a polynomial equation in $d^{*}, \cos \left(\theta^{*}\right)$, and $\sin \left(\theta^{*}\right)$ by requiring the numerator to be equal to zero. Therefore, computing all critical points of $\Pi_{3}$ is equivalent to solving the polynomial system defined by (28)-(29).

Similar to the distance-only problem, we examine two cases: (i) the constraint (27) is inactive, i.e., $g_{\beta}\left(d^{*}, \theta^{*}\right)<$ 0 ; (ii) the constraint is active, i.e., $g_{\beta}\left(d^{*}, \theta^{*}\right)=0$, and determine the global optimal solution by searching for the critical point that has the smallest objective function value.

1) Inactive constraint: We first compute the set of all stationary points, denoted as $\boldsymbol{\Xi}_{u}$, by assuming that the constraint is inactive, or equivalently,

$$
g_{\beta}\left(d^{*}, \theta^{*}\right)<0, \quad \forall\left(d^{*}, \theta^{*}\right) \in \boldsymbol{\Xi}_{u} .
$$

Combining (31) and the complementary slackness condition (29), yields $\lambda^{*}=0$. Hence, the term $\lambda^{*} \nabla g_{\beta}\left(d^{*}, \theta^{*}\right)$ in (28) vanishes. Thus, (28) and (29) are simplified into

$$
\nabla f_{\beta}\left(d^{*}, \theta^{*}\right)=\left[\begin{array}{l}
\nabla_{d} f_{\beta}\left(d^{*}, \theta^{*}\right) \\
\nabla_{\theta} f_{\beta}\left(d^{*}, \theta^{*}\right)
\end{array}\right]=\left[\begin{array}{l}
0 \\
0
\end{array}\right] \text {. }
$$

As shown in [15], (32) is equivalent to a system of two multivariate polynomial equations in variables $d^{*}$, $\cos \left(\theta^{*}\right)$, and $\sin \left(\theta^{*}\right)$. The first polynomial, derived from $\nabla_{d} f_{\beta}\left(d^{*}, \theta^{*}\right)=0$ and denoted as $\mathcal{F}_{d}$, has degree three; whereas the second polynomial $\mathcal{F}_{\theta}$, obtained through $\nabla_{\theta} f_{\beta}\left(d^{*}, \theta^{*}\right)=0$, is of degree four. 
In order to solve $\mathcal{F}_{d}=\mathcal{F}_{\theta}=0$, we first treat $\cos \left(\theta^{*}\right)$ and $\sin \left(\theta^{*}\right)$ as parameters and rewrite the $\mathcal{F}_{d}$ and $\mathcal{F}_{\theta}$ polynomials in $d^{*}$ as [15]

$$
\begin{aligned}
& \mathcal{F}_{d}=\zeta_{2}\left(d^{*}\right)^{2}+\zeta_{1} d^{*}+\zeta_{0} \\
& \mathcal{F}_{\theta}=\gamma_{3}\left(d^{*}\right)^{3}+\gamma_{2}\left(d^{*}\right)^{2}+\gamma_{1} d^{*}+\gamma_{0},
\end{aligned}
$$

where $\zeta_{i}, i=0,1,2$ and $\gamma_{j}, j=0, \ldots, 3$ are bivariate polynomials in $\cos \left(\theta^{*}\right)$ and $\sin \left(\theta^{*}\right)$ [15].

We first eliminate the variable $d^{*}$ using the Sylvester resultant [16, Ch. 3]. The Sylvester matrix of $\mathcal{F}_{d}$ and $\mathcal{F}_{\theta}$ with respect to $d^{*}$, denoted as $\operatorname{Syl}\left(\mathcal{F}_{d}, \mathcal{F}_{\theta} ; d^{*}\right)$, is the following $5 \times 5$ matrix

$$
\operatorname{Syl}\left(\mathcal{F}_{d}, \mathcal{F}_{\theta} ; d^{*}\right)=\left[\begin{array}{lllll}
\zeta_{2} & & & \gamma_{3} & \\
\zeta_{1} & \zeta_{2} & & \gamma_{2} & \gamma_{3} \\
\zeta_{0} & \zeta_{1} & \zeta_{2} & \gamma_{1} & \gamma_{2} \\
& \zeta_{0} & \zeta_{1} & \gamma_{0} & \gamma_{1} \\
& & \zeta_{0} & & \gamma_{0}
\end{array}\right]
$$

The resultant of $\mathcal{F}_{d}$ and $\mathcal{F}_{\theta}$ with respect to $d^{*}$, denoted as $\operatorname{Res}\left(\mathcal{F}_{d}, \mathcal{F}_{\theta} ; d^{*}\right)$, is the determinant of the Sylvester matrix $\operatorname{Syl}\left(\mathcal{F}_{d}, \mathcal{F}_{\theta} ; d^{*}\right)$. Furthermore, since $\zeta_{i}, i=0,1,2$ and $\gamma_{j}$, $j=0, \ldots, 3$ are bivariate polynomials of $\cos \left(\theta^{*}\right)$ and $\sin \left(\theta^{*}\right), \operatorname{Res}\left(\mathcal{F}_{d}, \mathcal{F}_{\theta} ; d^{*}\right)$ is also a bivariate polynomial of $\cos \left(\theta^{*}\right)$ and $\sin \left(\theta^{*}\right)$ :

$$
\mathcal{R}_{u}\left(\cos \left(\theta^{*}\right), \sin \left(\theta^{*}\right)\right):=\operatorname{Res}\left(\mathcal{F}_{d}, \mathcal{F}_{\theta} ; d^{*}\right)=0 .
$$

Together with the trigonometric identity

$$
\mathcal{I}:=\cos ^{2}\left(\theta^{*}\right)+\sin ^{2}\left(\theta^{*}\right)-1=0,
$$

we employ the Sylvester resultant again to eliminate one of the variables, $\cos \left(\theta^{*}\right)$, to obtain a univariate polynomial in $\sin \left(\theta^{*}\right)$ :

$$
\mathcal{S}_{u}:=\operatorname{Res}\left(\mathcal{R}_{u}, \mathcal{I} ; \cos \left(\theta^{*}\right)\right)=\sum_{j=0}^{11} \delta_{j} \sin ^{2 j}\left(\theta^{*}\right)=0
$$

where $\delta_{j}, j=0, \ldots, 11$ are known coefficients expressed in terms of $\mathbf{P}, d_{o}$, and $\sigma_{\beta}^{2}$. Note that (37) does not contain odd-degree terms of $\sin \left(\theta^{*}\right)$. Therefore, (37) is equivalent to the following eleventh-order univariate polynomial in the variable $s:=\sin ^{2}\left(\theta^{*}\right)$

$$
\mathcal{S}_{u}(s)=\sum_{j=0}^{11} \delta_{j} s^{j}=0 .
$$

The roots of the univariate polynomial $\mathcal{S}_{u}(s)$ correspond to the 11 eigenvalues of the associated $11 \times 11$ companion matrix $\Delta[17]$ :

$$
\boldsymbol{\Delta}=\left[\begin{array}{cccc}
0 & & & -\delta_{0} / \delta_{11} \\
1 & 0 & & -\delta_{1} / \delta_{11} \\
& \ddots & & \vdots \\
& & 1 & -\delta_{10} / \delta_{11}
\end{array}\right]
$$

Note that since $s=\sin ^{2}\left(\theta^{*}\right)$, we only need to consider the real solutions between 0 and 1 of (38). Once $s$ is determined, both $\cos \left(\theta^{*}\right)$ and $\sin \left(\theta^{*}\right)$ can be computed using the trigonometric identity, which can have at most 4 solutions for $\theta^{*}$. Finally, for each $\theta^{*}$, we compute the coefficients $\zeta_{j}, j=0,1,2$ [see (33)], and solve for $d^{*}$ from (33), which can have at most 2 solutions. Thus, the set $\boldsymbol{\Xi}_{u}$ consisting of all critical points $\left(d^{*}, \theta^{*}\right)$, has at most 88 elements. However, we only need to consider those critical points satisfying the constraint $g\left(d^{*}, \theta^{*}\right)<0$.

The next step is to evaluate the objective function $f_{\beta}(d, \theta)$ [see (26)] at all the points in $\boldsymbol{\Xi}_{u}$ and select the one with the smallest objective value as the global optimal solution of $\Pi_{3}$, for the case when the constraint is inactive. We refer to this optimal solution as $\left(d_{u}^{*}, \theta_{u}^{*}\right)$.

2) Active constraint: When the constraint is active, i.e., $g_{\beta}\left(d^{*}, \theta^{*}\right)=0$, the complementary slackness condition (29) is automatically satisfied. Hence, (28) and (29) are simplified into

$$
\begin{aligned}
\nabla f_{\beta}\left(d^{*}, \theta^{*}\right)+\lambda^{*} \nabla g_{\beta}\left(d^{*}, \theta^{*}\right) & =\mathbf{0}_{2 \times 1} \\
g_{\beta}\left(d^{*}, \theta^{*}\right) & =0 .
\end{aligned}
$$

We solve this system of equations by an elimination procedure similar to the inactive constraint case. The difference is that an additional variable $\lambda^{*}$ needs to be eliminated first. In order to do so, we multiply both sides of (39) with $\left(\nabla g_{\beta}\left(d^{*}, \theta^{*}\right)\right)^{T} \mathbf{J}$, where $\mathbf{J}=\mathbf{C}(-\pi / 2)$ and $\mathbf{C}(\cdot)$ is the $2 \times 2$ rotation matrix, and obtain a polynomial in only $d^{*}$, $\cos \left(\theta^{*}\right)$, and $\sin \left(\theta^{*}\right)$ :

$$
\mathcal{Q}\left(d^{*}, \cos \left(\theta^{*}\right), \sin \left(\theta^{*}\right)\right)=0 .
$$

Combining (41) with (40) and the trigonometric identity (36), we conclude that the set of all stationary points, denoted as $\boldsymbol{\Xi}_{c}$, must satisfy

$$
\begin{aligned}
\mathcal{Q}\left(d^{*}, \cos \left(\theta^{*}\right), \sin \left(\theta^{*}\right)\right) & =0 \\
g_{\beta}\left(d^{*}, \cos \left(\theta^{*}\right), \sin \left(\theta^{*}\right)\right) & =0 \\
\mathcal{I}\left(\cos \left(\theta^{*}\right), \sin \left(\theta^{*}\right)\right) & =0 .
\end{aligned}
$$

To solve the above system of polynomial equations, we employ the same strategy outlined in the inactive constraint case. Firstly, we use the Sylvester resultant to eliminate $d^{*}$ from (42) and (43) and obtain an eighth-order bivariate polynomial $\mathcal{R}_{c}\left(\cos \left(\theta^{*}\right), \sin \left(\theta^{*}\right)\right)$. Secondly, we eliminate $\cos \left(\theta^{*}\right)$ from $\mathcal{R}_{c}$ and $\mathcal{I}$, and arrive at a univariate polynomial in $\sin \left(\theta^{*}\right)$

$$
\mathcal{S}_{c}\left(\sin \left(\theta^{*}\right)\right)=\sum_{j=0}^{8} \eta_{j} \sin ^{2 j}\left(\theta^{*}\right)=0
$$

where $\eta_{j}, j=0, \ldots, 8$, are known coefficients expressed in terms of $\mathbf{P}, \mathbf{p}_{o}, r, d_{o}$, and $\sigma_{\beta}^{2}$. Since only even-degree terms appear in the above equation, it is equivalent to the following eighth-order univariate polynomial in $s:=\sin ^{2}\left(\theta^{*}\right)$

$$
\mathcal{S}_{c}(s)=\sum_{j=0}^{8} \eta_{j} s^{j}=0 .
$$

Similar to the inactive constraint case, the roots of (46) can be calculated by computing the eigenvalues of its associated $8 \times 8$ companion matrix. Once $s$ is determined, both $\cos \left(\theta^{*}\right)$ and $\sin \left(\theta^{*}\right)$ can be computed, which give at most 4 solutions for $\theta^{*}$. Finally, for each $\theta^{*}$, we solve for $d^{*}$ from (40), which can have at most 2 solutions. Thus, the set $\boldsymbol{\Xi}_{c}$ consisting of all critical points $\left(d^{*}, \theta^{*}\right)$, has at most 64 elements.

The final step is to evaluate the objective function $f_{\beta}(d, \theta)$ [see (26)] at all the critical points in $\boldsymbol{\Xi}_{c}$ and select the one with the smallest objective value as the global optimal solution of $\Pi_{3}$, for the case when the constraint is active. We refer to this optimal solution as $\left(d_{c}^{*}, \theta_{c}^{*}\right)$. 
3) Globally optimal solution for $\Pi_{3}$ : Finally, the globally minimal solution $\left(d_{\text {opt }}, \theta_{\text {opt }}\right)$ for $\Pi_{3}$ can be selected as

$$
\left(d_{\text {opt }}, \theta_{\text {opt }}\right)=\left\{\begin{array}{ccc}
\left(d_{u}^{*}, \theta_{u}^{*}\right) & : & f_{\beta}\left(d_{u}^{*}, \theta_{u}^{*}\right) \leq f_{\beta}\left(d_{c}^{*}, \theta_{c}^{*}\right) \\
\left(d_{c}^{*}, \theta_{c}^{*}\right) & : & f_{\beta}\left(d_{u}^{*}, \theta_{u}^{*}\right)>f_{\beta}\left(d_{c}^{*}, \theta_{c}^{*}\right)
\end{array}\right.
$$

\section{Simulation Results}

We have evaluated the performance of our motion strategy for a leader-follower formation in simulation. In particular, we consider the case where the leader moves on a straight line and the follower is supposed to maintain its position at $\mathbf{p}_{o}$ with respect to the leader. We have compared our motion strategy, the relaxed algebraic method (RAM), with four other strategies; namely (i) the "maintaining the formation" (MTF) strategy; (ii) a constrained random motion (CRM); (iii) the active control strategy of Mariottini et al. [10] (MME); and (iv) grid-based search (GBS). The strategy MTF always moves the follower toward the desired position $\mathbf{p}_{o}$, while CRM moves the follower to a random position inside a circle centered at $\mathbf{p}_{o}$ with radius $r$. For GBS, the area inside the circle is discretized into cells, and the follower moves to the cell that has the lowest cost. GBS should be a benchmark for evaluating the performance of all motion strategies, if the discrete cell size is sufficiently small.

We have conducted Monte Carlo simulations with 50 trials with the following settings. The desired follower position is $\mathbf{p}_{o}=[-1,-2]^{T}$ for the robot with range measurements, and $\mathbf{p}_{o}=[-1,2]^{T}$ for the robot with bearing measurements. The radius of the constrained circle is set to $r=0.1\left\|\mathbf{p}_{o}\right\|_{2}$. The initial estimated pose of the follower is $\hat{\mathbf{x}}_{1 \mid 1}=\left[\mathbf{p}_{o}^{T}, 0\right]^{T}$, with covariance $\mathbf{P}_{1 \mid 1}=\operatorname{diag}\left(0.1,0.1,4 \times 10^{-4}\right)$. We use the discrete-time kinematic model (9) with $\delta t=0.05 \mathrm{~s}$ to simulate the leader and follower motions. The leader's linear velocity is set to $1 \mathrm{~m} / \mathrm{s}$, with rotational velocity $0 \mathrm{rad} / \mathrm{s}$. The follower's control inputs, the linear and rotational velocities $v_{F m}$ and $\omega_{F m}$, are generated according to the next timestep position $\mathbf{p}$ determined by the specific motion strategy. In order to drive to $\mathbf{p}$, the follower first rotates with velocity $\omega_{F m}$, such that its heading points to $\mathbf{p}$. Then it executes a pure translation with velocity $v_{F m}$ to finally arrive at $\mathbf{p}$. The true follower's linear and rotational velocities are affected by zero-mean white Gaussian noise with standard deviation $\sigma_{v}=0.2 \mathrm{~m} / \mathrm{s}$ and $\sigma_{\omega}=0.1 \mathrm{rad} / \mathrm{s}$, respectively. At $\mathbf{p}$, the follower records a distance or bearing measurement to the leader, so as to update its pose estimate.

Fig. 4 shows the trajectories of RAM, GBS, and MME with one robot measuring distance to the leader and another robot measuring its bearing to the leader. The trajectories of MTF and CRM are not informative and therefore are omitted. RAM generates a zigzagging motion pattern which touches the circular constraint most of the time, while GBS also shows a similar motion pattern. The trajectory of MME is more irregular with larger position uncertainty depicted by the $3 \sigma$ ellipses.

\section{A. Distance-only Case}

Fig. 5 shows the follower's pose uncertainty of the aforementioned five motion strategies with distance measurement noise standard deviation $\sigma_{d}=0.1 \mathrm{~m}$. The metric used for evaluating uncertainty is the weighted trace of the posterior
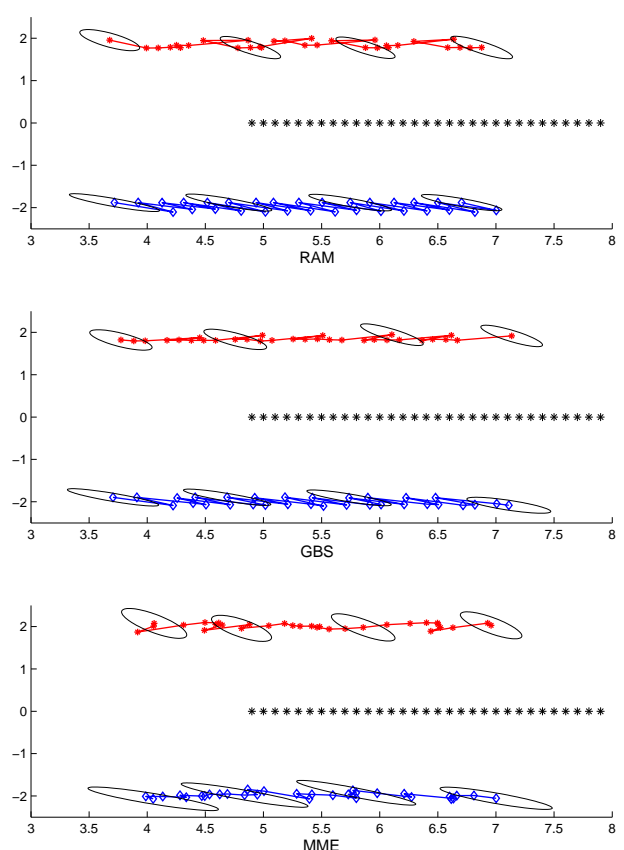

Fig. 4. The estimated trajectories of the two followers and the leader, when employing RAM, GBS, or MME as motion strategy. In each subplot, the follower equipped with a bearing sensor is on the top, the leader is in the middle, and the follower equipped with a range sensor is at the bottom. The ellipses denote the $3 \sigma$ bounds for the follower's position uncertainty at the corresponding positions.

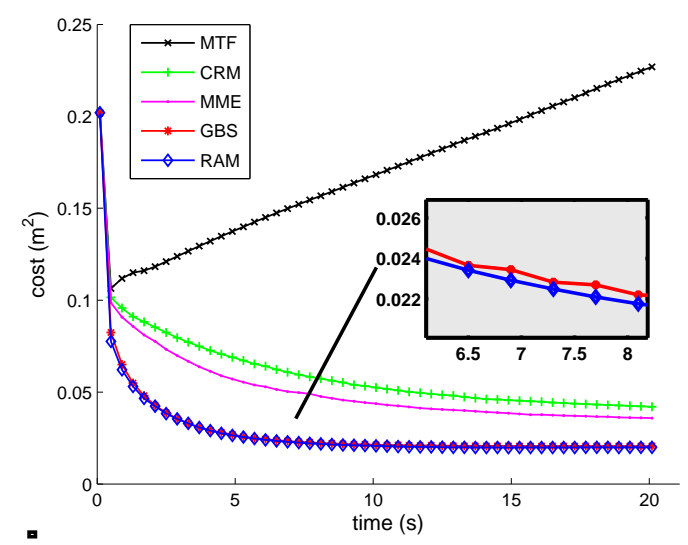

Fig. 5. Distance-only case, Monte Carlo simulations: Average weighted trace of the follower's posterior covariance matrix in 50 experiments.

covariance matrix (see Section III-D). Since the relative pose is unobservable when the follower moves in exact formation, the uncertainty of MTF increases unbounded over time. When the follower moves around the desired position, the relative pose becomes observable, which is demonstrated by the convergence of the uncertainty to almost steadystate values for the other four motion strategies. However, the specific motion strategy employed affects the estimate's uncertainty. CRM shows the highest uncertainty, closely followed by the MME strategy. This result is expected, because 


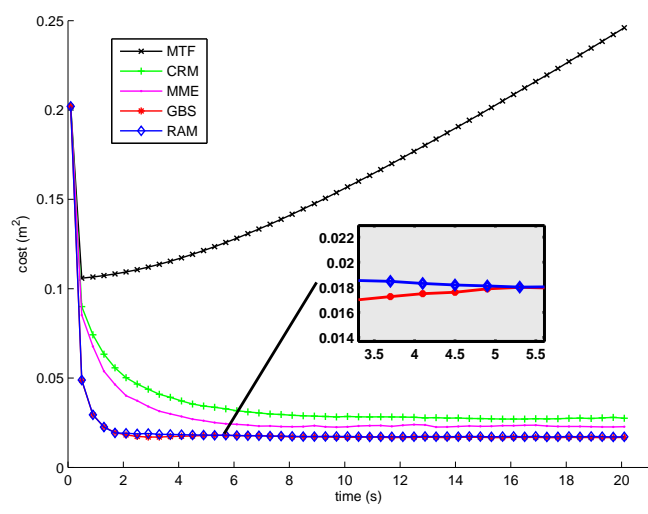

Fig. 6. Bearing-only case, Monte Carlo simulations: Average weighted trace of the follower's posterior covariance matrix in 50 experiments.

these two strategies are designed to gain observability, rather than minimize the uncertainty. Our motion strategy, RAM, is designed to minimize uncertainty and shows superior performance compared to CRM and MME. From the zoomin view of the uncertainty curves of RAM and GBS, we can see that our strategy is even slightly better than the benchmark strategy GBS. This is due to the fact that given limitations on how small the grid-cell size may be, the GBS may not reach the global minimum.

\section{B. Bearing-only Case}

We hereafter examine the performance of the RAM motion strategy for the bearing-only measurement model. In our simulation, the standard deviation of the bearing measurement noise is set to $\sigma_{\beta}=0.02 \mathrm{rad}$.

The time evolution of the weighted trace of the follower's posterior covariance matrix (i.e., the cost function) averaged over 50 experiments is shown in Fig. 6. As expected, the performance of RAM and GBS is improved compared to the cases of CRM and MME, and is significantly better than that of the unobservable case MTF. Additionally, the uncertainty in the follower's pose estimates (weighted trace of the covariance matrix) achieved by the proposed RAM motion strategy is indistinguishable of that of the GBS.

\section{CONCLUSION AND FUTURE WORK}

In this paper, we present a new approach to generate optimal motion strategies for a leader-follower formation. Specifically, we consider the case where the follower measures the distance or bearing to the leader, while the leader is moving on a straight line. Since the follower's pose is unobservable if it maintains a constant position with respect to the leader, we allow the follower to slightly deviate from its desired position, and determine its optimal next time-step position. In particular, we minimize the weighted trace of the posterior covariance matrix, and compute the global optimal solution analytically. Even though we focus on the case where the leader is moving on a straight line, our optimization method can be applied to arbitrary leader motions. The simulation results demonstrate superior localization accuracy compared to existing follower motion strategies, while showing indistinguishable performance comparing to a grid-based search strategy.

As part of our future work, we plan to investigate the case where the robots have access to vector measurements. In this case, the optimization problem becomes significantly more challenging, due to the trade-off between different sources of information. In addition, we are also interested in solving for optimal follower positions for more than one time step. Optimizing over a longer time horizon is expected to further increase the localization accuracy.

\section{REFERENCES}

[1] J. Bender, "An overview of systems studies of automated highway systems," IEEE Transactions on Vehicular Technology, vol. 40, no. 1, pp. 82-99, Feb. 1991.

[2] J. Spletzer, A. Das, R. Fierro, C. Taylor, V. Kumar, and J. Ostrowski, "Cooperative localization and control for multi-robot manipulation," in Proc. IEEE/RSJ International Conference on Intelligent Robots and Systems, Maui, HI, Oct. 29- Nov. 3 2001, pp. 631-636.

[3] T. Balch and R. Arkin, "Behavior-based formation control for multirobot teams," IEEE Transactions on Robotics and Automation, vol. 14, no. 6, pp. 926-939, Dec. 1998.

[4] K.-H. Tan and M. Lewis, "Virtual structures for high-precision cooperative mobile robotic control," in Proc. IEEE/RSJ International Conference on Intelligent Robots and Systems, Osaka, Japan, Nov. 4-8 1996, pp. 132-139.

[5] J. Desai, J. Ostrowski, and V. Kumar, "Controlling formations of multiple mobile robots," in Proc. IEEE International Conference on Robotics and Automation, Leuven, Belgium, May 16-20 1998, pp. 2864-2869.

[6] I. Rekleitis, G. Dudek, and E. Milios, "Multi-robot cooperative localization: a study of trade-offs between efficiency and accuracy," in Proc. IEEE/RSJ International Conference on Intelligent Robots and System, Lausanne, Switzerland, Sep. 30- Oct. 5 2002, pp. 2690-2695.

[7] N. Trawny and T. Barfoot, "Optimized motion strategies for cooperative localization of mobile robots," in Proc. IEEE International Conference on Robotics and Automation, New Orleans, LA, Apr. 26 - May 1 2004, pp. 1027-1032.

[8] A. Martinelli and R. Siegwart, "Observability analysis for mobile robot localization," in Proc. IEEE/RSJ International Conference on Intelligent Robots and Systems, Edmonton, Canada, Aug. 2-6 2005, pp. 1471-1476.

[9] X. S. Zhou and S. I. Roumeliotis, "Robot-to-robot relative pose estimation from range measurements," IEEE Transactions on Robotics, vol. 24, no. 6, pp. 1379-1393, Dec. 2008.

[10] G. L. Mariottini, S. Martini, and M. B. Egerstedt, "A switching active sensing strategy to maintain observability for vision-based formation control," in Proc. IEEE International Conference on Robotics and Automation, Kobe, Japan, May 12-17 2009, pp. 2637-2642.

[11] F. Zhang, B. Grocholsky, and V. Kumar, "Formations for localization of robot networks," in Proc. IEEE International Conference on Robotics and Automation, New Orleans, LA, Apr. 26-May 12004 , pp. 3369-3374.

[12] R. Kurazume and S. Hirose, "Study on cooperative positioning system: optimum moving strategies for CPS-III," in Proc. IEEE International Conference on Robotics and Automation, Leuven, Belgium, May 1620 1998, pp. 2896-2903.

[13] Y. Hidaka, A. Mourikis, and S. Roumeliotis, "Optimal formations for cooperative localization of mobile robots," in Proc. IEEE International Conference on Robotics and Automation, Barcelona, Spain, Apr. 1822 2005, pp. 4126-4131.

[14] D. P. Bertsekas, Nonlinear Programming, 2nd ed. Belmont, MA: Athena Scientific, 1999.

[15] K. Zhou and S. I. Roumeliotis, "Optimized motion strategy for bearing-only localization in leader-follower formations," MARS Lab. Dept. of Computer Science and Engineering, University of Minnesota, Tech. Rep., Mar. 2011, http://mars.cs.umn.edu/tr/reports/Ke11.pdf.

[16] D. A. Cox, J. B. Little, and D. O'Shea, Ideals, Varieties, and Algorithms: An Introduction to Computational Algebraic Geometry and Commutative Algebra, 3rd ed. New York: Springer, 2007.

[17] A. Edelman and H. Murakami, "Polynomial roots from companion matrix eigenvalues," Mathematics of Computation, vol. 64, no. 210, pp. 763-776, Apr. 1995. 